\title{
Optimización del proceso de eliminación de aloína del gel fileteado mecánico de Aloe vera concentrado 10X
}

\author{
Optimization of aloin removal process from the \\ mechanical filleted gel, Aloe vera concentrate10X
}

Walter J. Martínez B. Aloe Technology S.A.S-Universidad del Norte (Colombia) wmartinez@aloetechnology.com

\section{Milena Vélez Ramírez} Aloe Technology S.A.S-Universidad del Norte (Colombia)

Reynaldo Espitia P. Universidad del Norte (Colombia)
Carlos D. Paternina-Arboleda Universidad del Norte (Colombia)

\author{
Alexander Paroddy \\ Universidad Autónoma del Caribe \\ (Colombia) \\ Luis Paternina Tapia \\ Universidad de la Costa-CUC \\ (Colombia)
}

Revista Cumbres Vol.3 №2

Versión impresa ISSN 1390-9541

Versión electrónica ISSN 1390-3365

http://investigacion.utmachala.edu.ec/revistas/index.php/Cumbres 


\section{RESUMEN}

Se optimizó el proceso de eliminación de aloína del gel de Aloe vera en la planta de proceso Aloe Technology S.A.S, para ello se evaluaron tres variables de entrada: tiempo de agitación tA (30, 45 y $60 \mathrm{~min})$, temperatura $\left(40-70{ }^{\circ} \mathrm{C}\right)$ y concentración de carbón activado-CA (1,0; 1 ,5 y 2,0\%). La aloína se cuantificó por cromatografía líquida de alta eficacia. Los resultados muestraron que la concentración de CA y tA ejercen efecto significativo en la eliminación de la aloína del gel de Aloe vera, mientras que la temperatura no. Según los resultados obtenidos las condiciones óptimas para la eliminación de la aloína del gel son $1,5 \%$ de CA y tA de 45 min y una temperatura de $40^{\circ} \mathrm{C}$. El modelo encontrado tuvo un $\mathrm{R}^{2}=0,976$ y un $\mathrm{R}^{2}$ ajustado $=0,96$ lo cual indica que el modelo, explica con alta precisión el comportamiento de la concentración de aloína en el procesamiento del gel.

Palabras clave: Aloe vera, aloína, optimización, carbón activado.

\section{ABSTRACT}

We optimized the process for aloin removal from Aloe vera gel at the processing plant, Aloe Technology A.S. for this purpose, stirring time (30, 45 and 60 minutes), stirring temperature $\left(40\right.$ and $\left.70^{\circ} \mathrm{C}\right)$ and activated carbon concentration - CA (1.0, 1.5 and $2.0 \%$ ) were evaluated. The determination of aloin was carried out by HPLC. The results showed that the concentration of CA and tA had significant effect, while the temperature had no significant effect (at 95\% confidence level), in removing aloin from Aloe Vera gel. According to the results obtained, optimum conditions for eliminating gel aloin are $1.5 \%$ of CA and 45 min of tA and $40^{\circ} \mathrm{C}$ temperature. The model found has an $\mathrm{R}^{2}$ $=0.976$ and $\mathrm{R}^{2}$ Adjusted $=0.96$ indicating that the model explains with high precision the behavior of the concentration of aloin in gel processing.

Keywords: Aloe vera, aloin, optimization, activated carbon.

\section{INTRODUCCIÓN}

El Aloe vera comúnmente conocida como sábila es una planta tropical o subtropical caracterizada por las propiedades medicinales que le son atribuidas, es originaria de la península de Arabia (Vega et al., 2005; Ramachandra \& Rao, 2008). Desde el punto de vista medicinal, cosmetológico, terapéutico y alimenticio la parte más importante de la planta son sus alongadas y carnosas hojas que contienen en su interior lo que se conoce como gel de Aloe vera (Vega et al., 2005; Yun et al., 2009; Ramachandra \& Rao, 2008). Químicamente el gel está constituido en mayor proporción por agua, aproximadamente 98-99 \%, y el resto por ácidos galacturónicos, glucorónicos, mono, di y polisacáridos como glucosa, galactosa, arabinosa, fructosa, glucomanano, acemanano y manosa, esta última considerada por varios autores como el azúcar

\section{Cumbres}


más importante del gel. Además, otros tipos de sustancias como vitaminas, aminoácidos, compuestos fenólicos de gran poder antioxidante, como las cromonas y las antraquinonas que son los compuestos bioactivos mayoritarios (Mandal \& Das, 1980; T'hart et al., 1989; Pugh et al., 2001; He, et al., 2005; Bozzi et al., 2007).

A dichos compuestos les son atribuidas propiedades medicinales y terapéuticas por diferentes investigadores. Dixit \& Joshi (1983), encontraron que el Aloe vera reducía los niveles de colesterol. Gupta et al. (1981), Reynolds \& Dweck (1999), Vogler \& Ernst (1999) sugieren usar el gel de aloe para contrarrestar problemas de úlceras, gastrointestinales, estreñimiento, dolores de cabeza, artritis, deficiencias del sistema inmunológico, diabetes y gastroenteritis. De igual forma se ha encontraron que el Aloe vera puede ser empleado en tratamiento contra el cáncer, dado que inhibe el crecimiento de tumores (Dixit (1986), Wolfgang (1995), Eshum \& He (2004)).

Hoy día se sugiere emplear el gel de Aloe vera como materia prima en la elaboración de alimentos de características nutraceúticas, dados los compuestos funcionales contenidos en el gel (Loots et al., 2007; Lozano et al., 2011). Por otra parte, la cubierta externa de la hoja contiene sabia de color amarillento, siendo el principal componente de ese acíbar la Aloína con olor y sabor desagradables (Acurero, 2011; Domínguez et al., 2012). Según Rivero et al. (2002) la antraquinona es un potente laxante y por ello se debe minimizar hasta concentraciones permitidas o eliminar cuando el gel se va emplear para consumo directo o como materia prima para la fabricación de otros productos alimenticios. En este sentido la Unión Europea establece el límite máximo de concentración de aloína en productos destinados al consumo humano, siendo éste de 0,1mg/kg (CEE 88/388). Por esta razón, en el procesamiento del gel de Aloe vera se deben emplear medios y mecanismos para la eliminación de la aloína o reducirla hasta una concentración permitida. Este trabajo tuvo como objetivo determinar las condiciones óptimas de tiempo de agitación, temperatura y concentración de CA en el proceso de eliminación de aloína en el gel de Aloe vera (T3), además definir un modelo matemático que permita estimar el comportamiento de la concentración de aloína en el gel cuando varían las variables mencionadas.

\section{MATERIALES Y MÉTODOS}

Obtención del jugo de sábila/gel de Aloe vera: Se emplearon hojas de Aloe vera frescas de más de tres años de plantadas con longitud y masa mínima de $35 \mathrm{~cm}$ y $350 \mathrm{~g}$, respectivamente. Las hojas proceden de los cultivos ubicados en el corregimiento de Caracolí, municipio de Malambo (Departamento del Atlántico). Estas fueron lavadas y desinfectadas con Citrosan (3 ml /L), posteriormente se realizó la extracción y concentración hasta 10 X como se indica en la figura 1. Se empleó la enzima pectinasa de la casa comercial Novozymes, en una proporción de $25 \mathrm{ml} / \mathrm{T}$. El tratamiento se efectuó a 40 ${ }^{\circ} \mathrm{C}$ durante $2 \mathrm{~min}$. 


\section{Procesamiento de las hojas de Aloe vera (Planta Aloetechnology)}

En la Figura 1 se presenta el proceso optimizado y que fue empleado para la obtención del gel de Aloe vera y la eliminación de aloína. En este las hojas de Aloe vera se introducen en la fileteadora (FL) la cual separa la cáscara del cristal, este último enviado al tanque de agitación (T1) donde se realiza el tratamiento enzimático, seguidamente se hace pasar por la despulpadora para eliminar los restos de cáscara; parte del flujo se retorna al tanque de tratamiento (T1) para seguir aprovechando la enzima; y el resto dirigido al tanque de mezcla (T2) donde se inactiva la enzima. Posteriormente, el gel se concentra diez veces con respecto a su concentración inicial de sólidos solubles (SS), este parámetro es monitoreado en el punto MSS, en caso de no alcanzar la concentración deseada (10X) el gel se recirculaba. En el tanque (T3) se adiciona el carbón activado (CA), se realiza el tratamiento térmico con agitación a $80 \mathrm{rpm}$, durante un tiempo determinado. Seguidamente el gel es conducido por una por una batería de filtros en serie donde el carbón activado se retira del gel, en el punto muestreo (PM) se toman las muestras para monitorear el comportamiento de la concentración de aloína. Finalmente, en el tanque (T4) el gel se estabiliza adicionando ácido ascórbico, se pasteuriza (85-95 ${ }^{\circ} \mathrm{C}$ por $1-2 \mathrm{~min}$ ) en el intercambiador de calor (IC) y se envasa.

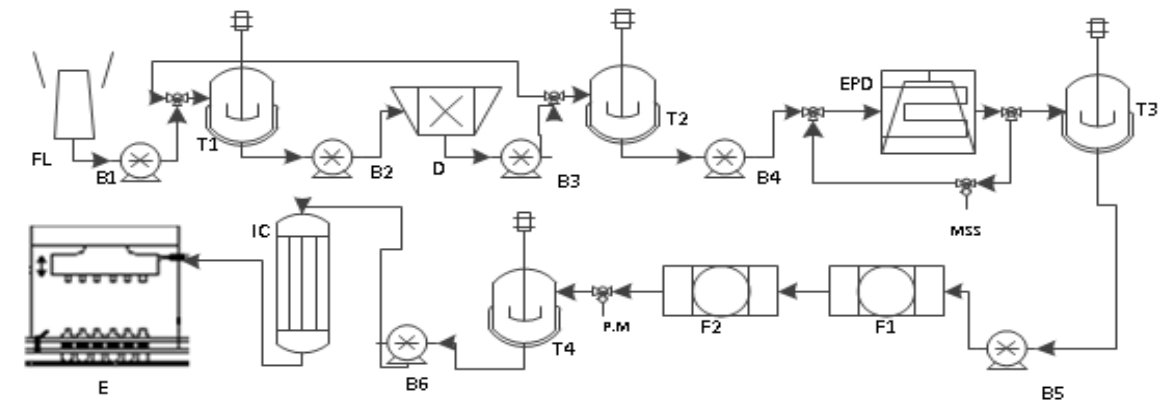

\begin{tabular}{|c|l|c|l|c|l|l|l|}
\hline $\mathbf{F L}$ & Fileteadora & $\mathbf{D}$ & Despulpadora & $\mathbf{F}_{\mathbf{1 , 2}}$ & Filtros prensa & $\mathbf{E}$ & Envasadora \\
\hline $\mathbf{B}_{1 . .}$ & $\begin{array}{l}\text { Bombas de } \\
\text { desplazamiento } \\
\text { positivo }\end{array}$ & $\mathbf{T}_{\mathbf{1 , 2 , 3 , 4}}$ & $\begin{array}{l}\text { Tanques de } \\
\text { agitación }\end{array}$ & $\mathbf{E P D}$ & $\begin{array}{l}\text { Evaporador de } \\
\text { pelicula } \\
\text { descendente }\end{array}$ & IC & $\begin{array}{l}\text { Intercambiador } \\
\text { de calor }\end{array}$ \\
\hline PM & \multicolumn{3}{|c|}{ Punto muestreo } & MSS & \multicolumn{2}{|c|}{ Monitoreo Sólidos Solubles } \\
\hline
\end{tabular}

Fig. 1: Proceso usado para la extracción del jugo/gel de Aloe vera

Eliminación de aloína: La eliminación de la aloína fue efectuada en el tanque encamisado con agitación (T3). La velocidad de agitación para la eliminación de la aloína permaneció constante a $80 \mathrm{rpm}$. En este proceso las variables manipuladas fueron temperatura, tiempo y concentraciones de CA. El tamaño de poro de los filtros empleados para retirar el CA fueron de 3 y $0,3 \mu \mathrm{m}$ para F1 y F2, respectivamente. Una vez finalizado el proceso de filtrado se tomó la muestra en PM y se determinó el contenido de aloína por cromatografía líquida de alta eficacia (CLAE).

Determinación de aloína: La determinación de aloína se llevó acabo según la metodología expuesta por Zonta et al. (1995) con algunas modificaciones. Se empleó estándar de aloína de la marca Sigma con pureza del 97\%, se realizó la curva patrón con 6 concentraciones $(0,1 ; 10 ; 20 ; 30 ; 40$ y 50 ppm),

\section{Cumbres}


obteniéndose un $\mathrm{R}^{2}=0,9921$. La preparación de los patrones fue realizada en una solución metanol y agua (50:50). La fase móvil utilizada consistió en una mezcla agua: acetonitrilo (20:80). Se realizó ensayo con una hoja de Aloe vera fresca con el fin de verificar la curva patrón. Para realizar la medición de las muestras se tomaron $3 \mu \mathrm{L}$ y se inyectaron directamente. El equipo empleado para la medición estaba compuesto de una bomba marca Hitachi L-2130Pump, Columna RP-18 (55 mm, 4mm (PUROSPHER ${ }^{\mathrm{TM}}$ ), $3 \mu \mathrm{m}$ ), detector Hitachi L-2420 UV $(\lambda=255)$.

Diseño experimental: Para optimizar el proceso se realizaron dos diseños experimentales sucesivos. El primero con el fin de determinar los factores y las interacciones significativas. Para dicho cribado se propuso un diseño 23 , todas las variables cuantitativas, con una réplica para un total de 16 experimentos. Los factores y los niveles establecidos fueron: concentración de CA (1,0 y 2,0\%), tiempo de agitación (30 y 60 min) y temperatura $\left(40^{\circ} \mathrm{C}\right.$ y $\left.70^{\circ} \mathrm{C}\right)$. El segundo diseño fue un $3 \mathrm{k}$ con cinco puntos centrales, donde $\mathrm{k}$ dependió de los parámetros que resultaron significativos en el primer diseño. Los resultados se analizaron mediante la metodología de superficie respuesta. El análisis de los resultados se desarrolló empleando el software Statgraphics V 16

\section{RESULTADOS Y DISCUSIÓN}

Los resultados obtenidos (Tabla 1) muestran que la concentración de aloína en el gel de Aloe vera disminuye hasta niveles no detectables cuando se usan concentraciones de CA del $2 \%$ y tiempos de agitación de 60 min, independientemente de la temperatura a la que se esté desarrollando el proceso. Sin embargo, ninguno de los tratamientos aplicados con 30 min de agitación fue suficiente para eliminar la antraquinona a niveles ND. Lo cual indica que el tiempo de contacto entre CA y la aloína en el proceso debe estar por encima de los 30 min. Según Mahajan et al. (1980), Güzel (1996) y Toso et al. (2006), el CA es el adsorbente más versátil y eficiente para remover compuestos, caracterizado por su mecanismo de adsorción que permite en este caso retener la aloína cuando entra en contacto con éste. La antraquinona se adhiere a la superficie para después ser eliminada junto con las partículas de CA en el proceso de filtrado.

Por otro lado, en los resultados obtenidos (Tabla 1 y Tabla 2) también se evidencia que usando 1\% CA aún quedan trazas detectables de aloína en el gel, lo cual es atribuible a la saturación del material adsorbente, es decir para obtener geles libres de aloína deben emplearse concentraciones superiores a esta.

Según Sotelo et al. (1999) la adsorción con CA es un fenómeno físico, lo cual permite la desorción del compuesto una vez que se ha agotado la capacidad del adsorbente, dado el carácter reversible de este tipo de proceso. De igual forma Orta et al. (2003) afirma que al aumentar la concentración de carbón activado aumenta directamente el área superficial de contacto, lo cual favorece la eliminación de la aloína. 
Tabla 1: Matriz del diseño $2^{3}$

\begin{tabular}{ccccc}
\hline $\begin{array}{c}\text { Orden de } \\
\text { ejecución }\end{array}$ & $\begin{array}{c}\mathbf{A} \\
\text { carbón } \\
\text { activado(\%p/v) }\end{array}$ & $\begin{array}{c}\text { B } \\
\text { tiempo } \\
\text { agitación }(\mathbf{m i n})\end{array}$ & $\begin{array}{c}\mathbf{C} \\
\text { temperatura } \\
\left({ }^{\circ} \mathbf{C}\right)\end{array}$ & $\begin{array}{c}\boldsymbol{Y}_{\boldsymbol{1}} \text { contenido } \\
\text { de aloína }(\mathbf{p p m})\end{array}$ \\
\hline $\mathbf{1}$ & 1 & 30 & 70 & 35,5 \\
$\mathbf{2}$ & 2 & 60 & 40 & 0,1 \\
$\mathbf{3}$ & 2 & 30 & 70 & 11,21 \\
$\mathbf{4}$ & 2 & 30 & 70 & 11,1 \\
$\mathbf{5}$ & 1 & 60 & 40 & 23,4 \\
$\mathbf{6}$ & 2 & 30 & 40 & 10,95 \\
$\mathbf{7}$ & 1 & 30 & 40 & 34,95 \\
$\mathbf{8}$ & 2 & 60 & 40 & 0,1 \\
$\mathbf{9}$ & 2 & 30 & 40 & 11,3 \\
$\mathbf{1 0}$ & 1 & 60 & 40 & 22,84 \\
$\mathbf{1 1}$ & 2 & 60 & 70 & 0,1 \\
$\mathbf{1 2}$ & 1 & 30 & 70 & 33,1 \\
$\mathbf{1 3}$ & 1 & 60 & 70 & 24,3 \\
$\mathbf{1 4}$ & 1 & 60 & 70 & 21,81 \\
$\mathbf{1 5}$ & 1 & 30 & 40 & 35,5 \\
$\mathbf{1 6}$ & 2 & 60 & 70 & 0,1 \\
\hline
\end{tabular}

*ND en este trabajo se asumió como "0,1"que fue la concentración más baja empleada en la curva de calibración. La concentración promedio inicial de aloína del gel concentrado (10X) fue de 46,5 \pm $0.032 \mathrm{ppm}$

Tabla 2: Matriz del diseño experimental $3^{3}$

\begin{tabular}{|c|c|c|c|c|}
\hline $\begin{array}{l}\text { orden de } \\
\text { ejecución }\end{array}$ & $\begin{array}{c}\text { A } \\
\text { carbón activado } \\
(\% p / v)\end{array}$ & $\begin{array}{c}\text { B } \\
\text { tiempo agitación } \\
(\mathrm{min})\end{array}$ & $\begin{array}{c}Y_{2} \\
\text { contenido de } \\
\text { aloína (ppm) }\end{array}$ & $\begin{array}{c}Y_{2}^{\prime} \text { Valores } \\
\text { predichos } \\
\text { (modelo) }\end{array}$ \\
\hline $\mathbf{1}$ & 1,5 & 45 & 0,1 & 0,78 \\
\hline 2 & 2 & 30 & 10,34 & 11,61 \\
\hline 3 & 1,5 & 45 & 0,1 & 0,78 \\
\hline 4 & 1,5 & 60 & 0,1 & $-0,16$ \\
\hline 5 & 1 & 45 & 28,67 & 24,73 \\
\hline 6 & 1,5 & 45 & 0,1 & 0,78 \\
\hline 7 & 1,5 & 45 & 0,1 & 0,79 \\
\hline 8 & 2 & 60 & 0,1 & $-1,00$ \\
\hline 9 & 1 & 60 & 22,41 & 23,78 \\
\hline 10 & 1,5 & 45 & 0,1 & 0,78 \\
\hline 11 & 1 & 30 & 33,83 & 36,40 \\
\hline 12 & 1,5 & 30 & 16,3 & 12,46 \\
\hline 13 & 1,5 & 45 & 0,1 & 0,78 \\
\hline 14 & 2 & 45 & 0,1 & $-0,06$ \\
\hline
\end{tabular}

El Análisis de varianza-ANOVA (Tabla 3) mostró que el \%CA y el tiempo de agitación ejercen efecto significativo $(p \leq 0,05)$, en la remoción de la aloína del gel, mientras que la temperatura no tuvo efecto significativo en los rangos ensayados. Es decir, un aumento en la temperatura no mejora la eliminación de la aloína y por ello en los ensayos posteriores se usó $40^{\circ} \mathrm{C}$ en el proceso de eliminación de la aloína del gel. Según Ramos et al.(2002) la capacidad de adsorción del CA se aumenta cuando disminuye el $\mathrm{pH}$ y con la adición de sales principalmente cloruro de sodio, más no por el aumento de la temperatura. Cabe resaltar que en el diseño 23 se encontraron condiciones que reducían el contenido de aloína hasta niveles ND, sin embargo, no hubo argumentos suficientes para asegurar que se trataba de un punto óptimo del proceso, por lo cual las curvas de nivel del diseño se usaron para escoger la dirección en la que se dirigió el trabajo (Humberto y Román, 2008).

De la misma forma en la figura se observa que CA y tiempo de agitación son las variables que ejercen efectos significativos sobre la concentración de aloína en el gel, la temperatura no mostró efecto alguno. 
Tabla 3. Análisis de varianza diseño $2^{3}$

\begin{tabular}{llllll}
\hline Efecto & $\boldsymbol{S S}$ & $\boldsymbol{G l}$ & $\boldsymbol{M S}$ & $\boldsymbol{F}$ & Valor-P \\
\hline $\begin{array}{l}\text { A:[ ] Carbón } \\
\text { Activado }\end{array}$ & 2170,86 & 1 & 2170,86 & 3004,83 & 0,0000 \\
B:t. Agitación & 515,177 & 1 & 515,177 & 713,09 & 0,0000 \\
C:Temperatura & 0,213906 & 1 & 0,213906 & 0,30 & 0,5996 \\
Agitación & & & & & \\
AB & 0,381306 & 1 & 0,381306 & 0,53 & 0,4860 \\
AC & 0,242556 & 1 & 0,242556 & 0,34 & 0,5765 \\
BC & 0,158006 & 1 & 0,158006 & 0,22 & 0,6511 \\
Error total & 6,50211 & 9 & 0,722456 & & \\
Total (corr.) & 2693,54 & 15 & & & \\
\hline
\end{tabular}

Los resultados obtenidos en el diseño 32 mostraron la misma tendencia (Tabla 2). El ANOVA (Tabla 4) reafirma que tanto la concentración de carbón activado como el tiempo de agitación tienen efecto significativo $(p \leq 0,05)$ en la eliminación de aloína. También se encontró que el cuadrado de cada factor tiene efectos significativos, mas no la interacción de estos. Dicho comportamiento se evidencia de forma más clara en la superficie de contorno del diseño 32 (Figura 3).

Tabla 4. Análisis de varianza diseño $3^{2}$

\begin{tabular}{llllll}
\hline Efecto & $\boldsymbol{S S}$ & $\boldsymbol{G l}$ & $\boldsymbol{M S}$ & $\boldsymbol{F}$ & Valor-P \\
\hline $\begin{array}{l}\text { A:Carbon } \\
\text { activado }\end{array}$ & 921,816 & 1 & 921,816 & 166,85 & 0,0000 \\
$\begin{array}{l}\text { B:Tiempo } \\
\text { agitación }\end{array}$ & 238,897 & 1 & 238,897 & 43,24 & 0,0002 \\
AA & 377,878 & 1 & 377,878 & 68,40 & 0,0000 \\
AB & 0,3481 & 1 & 0,3481 & 0,06 & 0,8081 \\
BB & 81,5078 & 1 & 81,5078 & 14,75 & 0,0049 \\
Error total & 44,1975 & 8 & 5,52468 & & \\
Total (corr.) & 1938,13 & 13 & & & \\
\hline
\end{tabular}

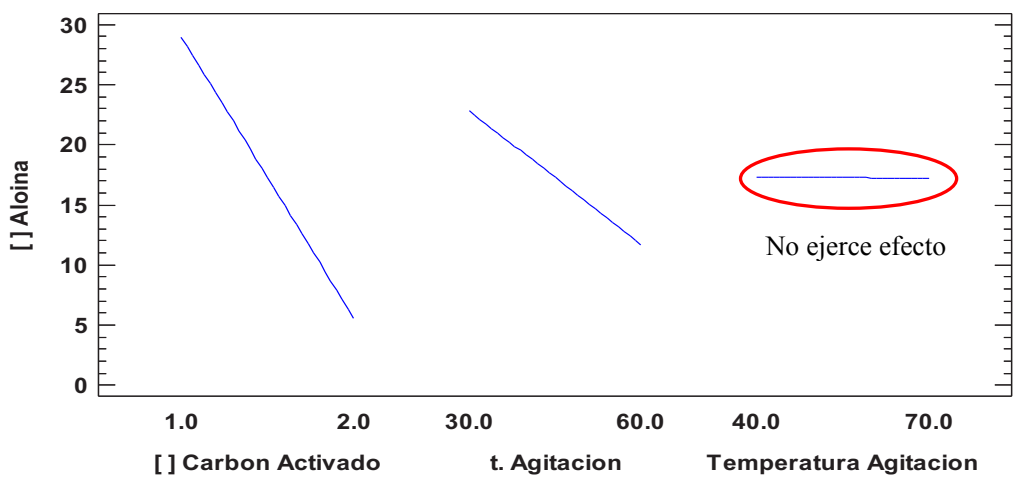

Fig 2. Variables que tienen efectos significativos sobre la concentración de aloína

En la gráfica también se observa que tiempos más cortos de agitación requieren mayor concentración de CA y viceversa, sin embargo condiciones con concentraciones de CA superiores al $2 \%$ no se ensayaron porque se incrementaba la frecuencia de cambio de los filtros "tiempo muerto" dado que deben retirar mayor cantidad de CA en el gel y por ende las telas filtrantes se sellan más rápido, condición que tampoco es favorable en el sistema por 
todo el tiempo que consume el ensamble, desensamble y la limpieza de la línea de proceso.

Por otro lado, tiempos de agitación superiores a los 60 min no fueron ensayados dado que también ocasionaban retrasos demasiados extensos en toda la línea de proceso. Los resultados obtenidos permitieron, mediante un análisis de regresión, estimar un modelo matemático que simula el comportamiento de la concentración de aloína en el gel de sábila cuando se manipularon las dos variables que resultaron significativas en dicho proceso

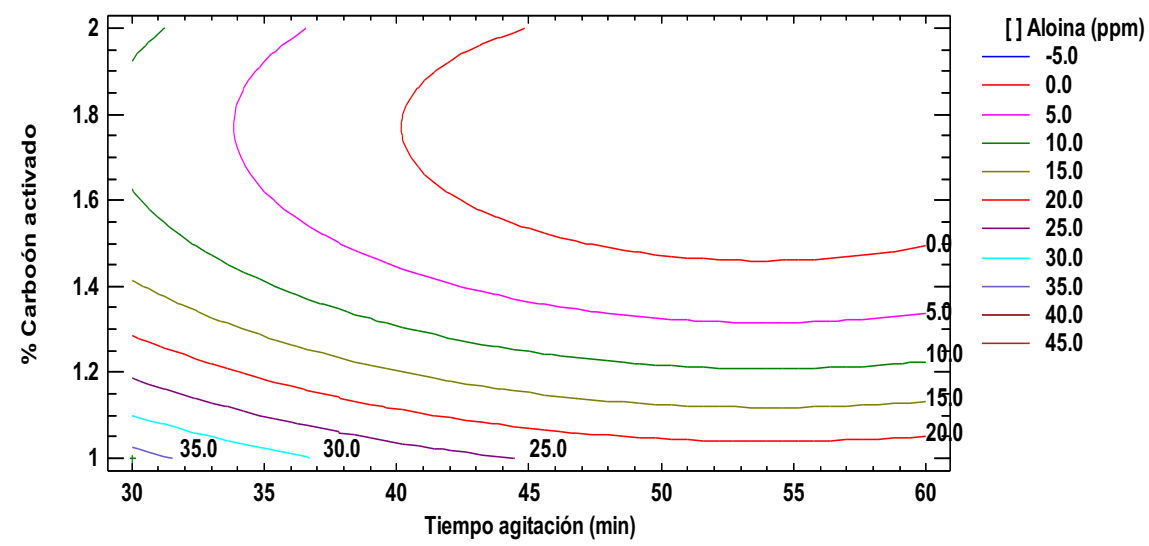

Fig. 3: Curvas de nivel diseño $3^{2}$

El modelo ajustado (Ecuación 1) mostró un $\mathrm{R}^{2}$ Ajustado $=0,96$ lo cual indica que el modelo tiene alta correlación, es decir explica $96 \%$ de la variabilidad de la aloína en el gel. Lo que indica que la concentración de aloína en el gel está gobernada mayoritariamente por las dos variables que resultaron significativas, mientras que el $4 \%$ restante es atribuible a factores externos no controlables.

[Aloina $]=209.108-163.372 * \mathrm{CA}-2.56608 * \mathrm{tA}+46.194 * \mathrm{CA}^{2}+0.0238379 * \mathrm{tA}^{2}$

(1)

CA: Carbón activado, tA: tiempo agitación

Finalmente, se corrió el modelo en los rangos ensayados e igualando la concentración de aloína a cero, encontrándose las condiciones óptimas para la eliminación de aloína (CA: 1,76\%; t: 40,24 min) que comparadas con las mejores condiciones experimentales (CA: 1,5\%; t: 45 min) están bastante próximas, dicho comportamiento también se evidencia en los valores experimentales y predichos del contenido de aloína (Tabla 2) y es consecuente según lo visto en la curvas de nivel (Figura 2). La proximidad entre los valores experimentales y predichos se comprobó por el bajo valor de los residuos, lo cual verifica el ajuste adecuado del modelo para predecir la variación de la variable dependiente como función de las variables CA y tA. En las Figuras 4 y 5 se muestran los cromatogramas para la mejor condición experimental y la óptima según el modelo respectivamente.

\section{Cumbres}




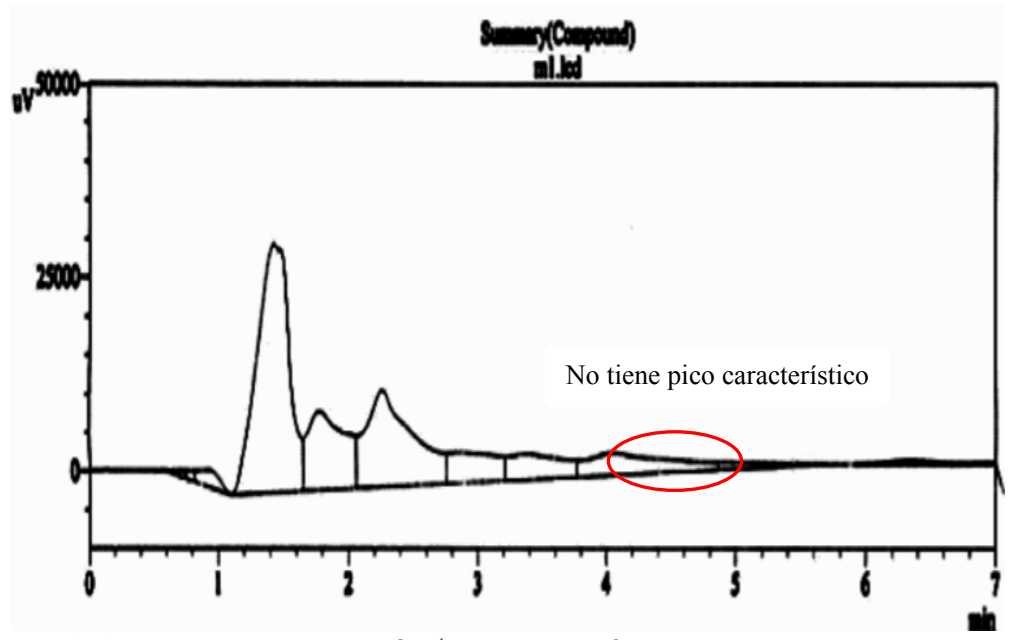

Fig. 4: Cromatograma mejor condición experimental

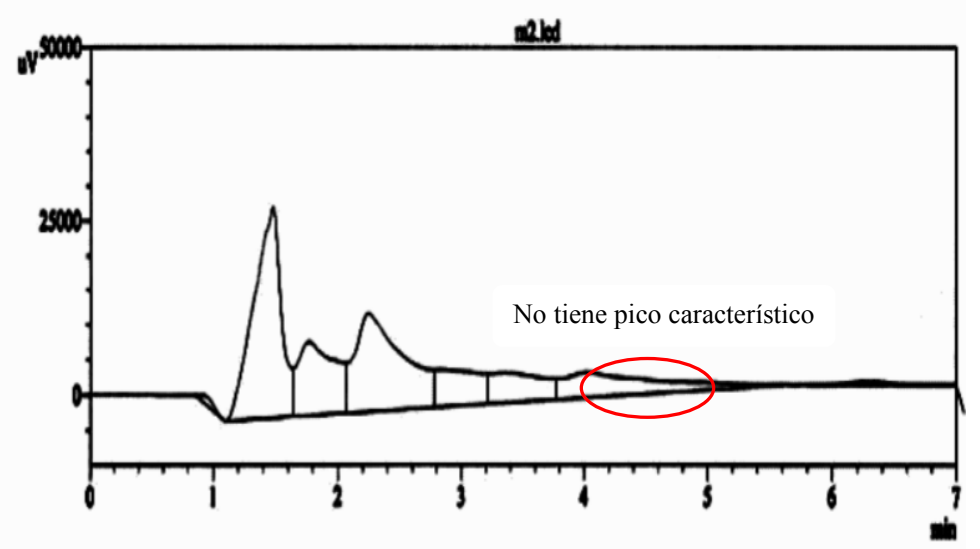

Fig. 5: Cromatograma condición optima según el modelo

En las gráficas no se aprecia el pico característico de la aloína, la cual tiene un tiempo de retención comprendido entre los 4 y $5 \mathrm{~min}$, esto indica que la concentración de aloína se encontraba en niveles muy bajos o ND. Sin embargo, para el caso práctico en la planta de proceso resulta más viable agitar cinco min más el gel que agregar 0,26 \% más de CA por las condiciones mencionadas anteriormente. Cabe destacar que la concentración óptima de CA determinada con este trabajo está por debajo a la empleada por Sierra (2002) cuando desarrolló jugos a base de sábila, la cual ascendió al 3\%.

\section{CONCLUSIONES}

Se optimizó la eliminación de aloína en la línea de procesamiento de gel de Aloe vera, controlando las variables CA, tiempo de agitación y la temperatura. Los ajustes realizados a las variables mencionadas permitieron reducir los costos de operación y aumentar los volúmenes de gel filtrado.

Se encontró un modelo matemático que explica el comportamiento del 
contenido de aloína en el gel cuando son manipuladas las variables de entradas usadas en este proceso.

\section{AGRDECIMIENTOS}

A la gobernación del Departamento del Atlántico por el financiamiento de esta investigación en el proyecto: Desarrollo Programa de desarrollo tecnológico agroindustrial y bioenergético del departamento del Atlántico Caribe, Atlántico, Todo El Departamento, Código BPIN No. 2013000100049, bajo contrato No. 0103*2013*000030.

\section{REFERENCIAS BIBLIOGRÁFICAS}

Acurero, Á. M. (2011). Producción de aloína en callos y hojas de brotes de zábila (Aloe vera L.) regenerados in vitro. Bioagro, 23(2), 79-87.

Bozzi, A., Perrin, C., Austin, S., \& Vera, F. A. (2007). Quality and authenticity of commercial aloe vera gel powders. Food chemistry, 103(1), 22-30.

Dixit, V. P. (1986). Hypolipidemic effect of Aloe barbadensis (Aloe fraction I) in cholesterol-fed rats. Lipid and Lipoprotein Metabolism. Proc. National Academy Science India: Sect. B (Biol. Sci.), 5, 339-342.

Domínguez-Fernández, R. N., Arzate-Vazquez, I., Chanona-Perez, J. J., Welti-Chanes, J. S., Alvarado-González, J. S., Calderon-Dominguez, G., ... \& Gutierrez-Lopez, G. F. (2012). El gel de Aloe vera: estructura, composición química, procesamiento, actividad biológica e importancia en la industria farmacéutica y alimentaria. Revista mexicana de ingeniería química, 11(1), 2343.

Eshun, K., \& He, Q. (2004). Aloe vera: a valuable ingredient for the food, pharmaceutical and cosmetic industries-a review. Critical reviews in food science and nutrition, 44(2), 91-96.

Gupta, M. B., Nath, R., Gupta, G. P., \& Bhargava, K. P. (1981). Antiulcer activity of some plant triterpenoids. The Indian journal of medical research, 73, 649-652.

Güzel, F. (1996). The effect of surface acidity upon the adsorption capacities of activated carbons. Separation science and technology, 31(2), 283-290.

He, Q., Changhong, L., Kojo, E., \& Tian, Z. (2005). Quality and safety assurance in the processing of Aloe vera gel juice. Food control, 16(2), 95-104.

Humberto G.P. y Roman V.S. 2008. Análisis y diseño de experimentos. McGraw-Hill, México

Loots, D. T., van der Westhuizen, F. H., \& Botes, L. (2007). Aloe ferox leaf gel phytochemical content, antioxidant capacity, and possible health benefits. Journal of agricultural and food chemistry, 55(17), 6891-6896.

Lozano, L. A. L., Nova, C. M., \& Uribe, L. D. M. (2011). Estabilización del gel de Aloe barbadensis Miller y disminución de su concentración por adsorción en columna con carbón activado. REVISTA ION, 24(1).

Mahajan, O. P., Moreno-Castilla, C., \& Walker Jr, P. L. (1980). Surface-treated

\section{Cumbres}


activated carbon for removal of phenol from water. Separation Science and Technology, 15(10), 1733-1752.

Mandal, G., \& Das, A. (1980). Structure of the glucomannan isolated from the leaves of Aloe barbadensis Miller. Carbohydrate research, 87(2), 249-256.

Ndhlala, A. R., Amoo, S. O., Stafford, G. I., Finnie, J. F., \& Van Staden, J. (2009). Antimicrobial, anti-inflammatory and mutagenic investigation of the South African tree aloe (Aloe barberae). Journal of ethnopharmacology, 124(3), 404-408.

Orta, M. M. H., Barrón, J. M., Coronado, R. M. G., \& Ramos, R. L. (2003). Adsorción de pentaclorofenol en solución acuosa sobre carbones activados comerciales. Revista internacional de contaminación ambiental, 19(3), 137-144.

Pugh, N., Ross, S. A., ElSohly, M. A., \& Pasco, D. S. (2001). Characterization of Aloeride, a new high-molecular-weight polysaccharide from Aloe vera with potent immunostimulatory activity. Journal of agricultural and food chemistry, 49(2), 1030-1034.

Ramachandra, C. T., \& Rao, P. S. (2008). Processing of Aloe vera leaf gel: a review. American Journal of Agricultural and Biological Sciences, 3(2), 502-510.

Ramos, R. L., Vargas, L. V., Barrón, J. M., \& Coronado, R. G. (2002). Adsorción de salicilato de sodio en solución acuosa sobre carbón activado. Revista de la sociedad Química de México, 46(2), 159-166.

Reynolds, T., \& Dweck, A. C. (1999). Aloe vera leaf gel: a review update. Journal of ethnopharmacology, 68(1), 3-37.

Rivero Martínez, R., Rodríguez Leyes, E. A., Menéndez Castillo, R., Fernández Romero, J. A., Barrio Alonso, G. D., \& González Sanabia, M. L. (2002). Obtención y caracterización preliminar de un extracto de Aloe vera L. con actividad antiviral. Revista Cubana de plantas medicinales, 7(1), 32-38.

Sierra A. 2002. Desarrollo de un prototipo de bebida de sábila (Aloe Vera barbadensis Miller) y naranja”. Tesis de grado Ingeniero Agrónomo. Escuela Agrícola Zamorano. Honduras.

Sotelo, J. L., Ovejero, G., Delgado, J. A., \& Martínez, I. (1999). Eliminación de compuestos organoclorados para potabilización de aguas mediante un proceso de adsorción-regeneración en carbón activado. Univ. Complutense de Madrid, España.

t’Hart, L. A., Van den Berg, A. J., Kuis, L., Van Dijk, H., \& Labadie, R. P. (1989) An anti-complementary polysaccharide with immunological adjuvant activity from the leaf parenchyma gel of Aloe vera. Planta medica 55(6): 509-512

Toso, J. P., Aja Muñiz, R., Vallone, A., Fernandez Rodriguez, A., Castillo, C. R., Sapag, K., \& Zgrablich, G. (2006, June). Influencia del Tiempo de Activación en el Desarrollo de la Porosidad de Carbones Activados Físicamente. In ANALES AFA (Vol. 18, No. 1).

Urbina, L. A. L., Nova, C. M., \& Uribe, L. D. M. (2011). Estabilización del gel de Aloe barbadensis Miller y disminución de su concentración por adsorción en columna con carbón activado. REVISTA ION, 24(1).

Vega, G., Nevenka Ampuero, C., Luis Diaz, N., \& Roberto Lemus, M. (2005). Aloe vera (aloe barbadensis miller) as a component of functional foods. $R E$ VISTA CHILENA DE NUTRICION, 32(3), 208. 
Vogler, B. K., \& Ernst, E. (1999). Aloe vera: a systematic review of its clinical effectiveness. Br J Gen Pract, 49(447), 823-828.

Wolfgang, W. (1995). Healing with aloe. Ennsthaler 44(1): 3-5

Yun, N., Lee, C. H., \& Lee, S. M. (2009). Protective effect of Aloe vera on polymicrobial sepsis in mice. Food and chemical toxicology, 47(6), 1341-1348.

Zonta, F., Bogoni, P., Masotti, P., \& Micali, G. (1995). High-performance liquid chromatographic profiles of aloe constituents and determination of aloin in beverages, with reference to the EEC regulation for flavouring substances. Journal of Chromatography A, 718(1), 99-106. 